\title{
Improving Triggering Characteristics for a Surface-Flashover Triggered Vacuum Switch
}

\author{
Z. He, L. Wang, F. Li, W. Yao, M. Dong and C. ZhaO \\ College of Electrical and Electronic Engineering, Huazhong University of Science and Technology \\ 1037, Luoyu Rd., Wuhan, 430074, China
}

\begin{abstract}
A surface-breakdown triggered vacuum switch with parallel disk electrodes and semiconductor surface flashover trigger electrode has many advantages, it is easy to be triggered and machined. The resistance of the trigger gap has been increased in terms of modifying the thickness of the semiconductor layer and improving coating process technology. The trigger gap resistance, trigger voltage and trigger waveforms were experimentally tested. The results show that the triggering characteristics can be improved by increasing the trigger resistance.
\end{abstract}

PACS numbers: $52.80 . \mathrm{Vp}, 52.75 . \mathrm{Kq}, 52.25 . \mathrm{Mq}$

\section{Introduction}

The triggered vacuum switch (TVS) is a versatile device which can be made to withstand a high voltage across a relatively short gap and in which a high current metal vapor arc can be established by applying a suitable pulse of voltage to a trigger electrode.

Raju et al. investigated the electrical characteristics of TVS with different dielectric materials in 1975 [1]. In 1976, they studied the electrical properties of TVS employing a dielectric coated with a semiconducting layer [2]. In their report the characteristics of the TVS is studied in low main gap current in the range of 50$5000 \mathrm{~A}$. As the demand accompanied the development of pulsed power technology, specifications and capability of TVS improved greatly. Our research is to improve the triggering characteristics of the TVS employing a semiconductor layer, and to research the variations of the trigger resistance and the trigger voltage in the high-current operating condition.

\section{The experiment setup}

Figure 1a shows the schematic structure of the electrodes of our typical triggered vacuum switch. The switch employs two parallel disk electrodes. The cathode contains a trigger pin and an insulating ceramic sleeve coated with a semiconductor layer. Both main electrodes are made of oxygen-free high-conductivity copper (OFHC). The sealed-off vacuum pressure is maintained below $1.33 \times 10^{-5} \mathrm{~Pa}$.

Two experimental TVS tubes termed TVS-1 and TVS-2 are used. For TVS-1, the initial resistance of the surface of dielectric forming the trigger gap is $500 \Omega$. TVS-2 has the higher resistance, the initial value is

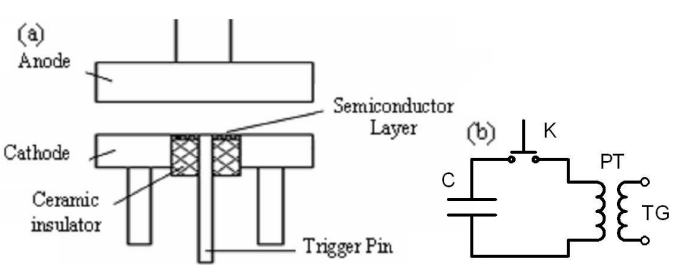

Fig. 1. (a) Schematic structure of the electrodes of TVS. (b) The triggering circuit of TVS. PT: Pulse Transformer, TG: Trigger gap.

$200 \mathrm{M} \Omega$. We increase the initial resistance of the trigger gap by modifying the thickness of the semiconductor layer and improving coating process technology.

The TVSs are tested up to $13 \mathrm{kV}$ charging voltage, $100 \mathrm{kA}$ peak current and $300 \mu \mathrm{s}$ current pulse-width. Figure $1 \mathrm{~b}$ shows the triggering circuit of TVS. The capacitor $\mathrm{C}$ is $40 \mu \mathrm{F}$ and charging voltage is $300 \mathrm{~V}$. Pulse transformer non-load output voltage is $28 \mathrm{kV}$.

\section{Experimental results and discussion}

For TVS-1, the initial resistance of the trigger gap is about $500 \Omega$ and the value is found to vary after every high current experiment. Figure 2 shows the dependence of the trigger gap resistance and the trigger voltage on the number of firings.

It shows that the resistance increases in the initial stage of the experiments. As the number of firings increases the resistance fluctuates between $800 \Omega$ and $1100 \Omega$. When the firing number of times exceeds 20 , the resistance of the trigger gap decreases. The resistance drops to $135 \Omega$ at the end of the experiments (see Fig. 2a). The variation of the trigger voltage and the resistance are almost identical (see Fig. 2b). It will be 


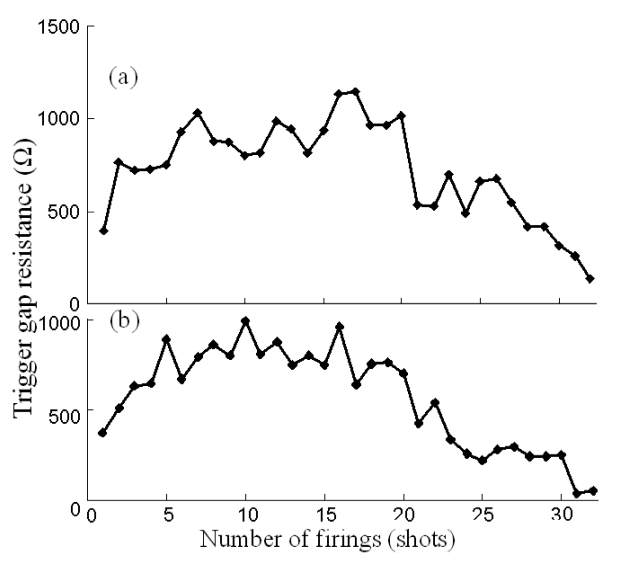

Fig. 2. (a) Variation of trigger gap resistance with number of firings. (b) Variation of trigger voltage with number of firings.

observed from Fig. 2 that the higher the trigger resistance, the higher the trigger voltage.

We suggest that the variation of the resistance is the consequence of the equilibrium between the deposition of the metal vapor and the evaporation of the semiconductor material. The main gap discharge releases metal vapor into the gap. A proportion of metal vapor is deposited on the dielectric surface, and the deposition would decrease the resistance. The temperature of the metal vapor is so high that the surrounding semiconductor is heated and evaporates. Because of the loss of the semiconductor, the evaporation would increase the resistance. Figure 3 shows the scattered metal particles on the surface of the semiconductor layer.

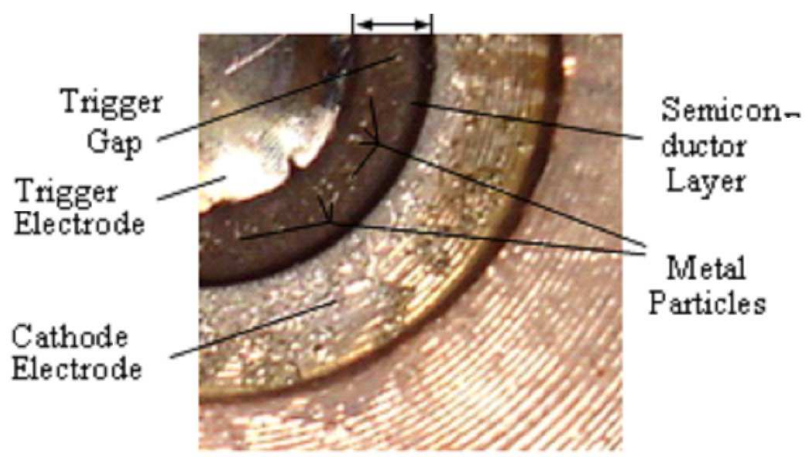

Fig. 3. Photo demonstrating metal particles on the surface of the semiconductor layer.

At the beginning of the high current experiments, the deposition of the metal vapor almost can be ignored, and the evaporation of the semiconductor material plays the leading role. It results in the rising of the resistance.

For the next stage, a larger amount of vapor is produced and therefore the deposition of metal is in equilibrium with the evaporation of the semiconductor. The resistance fluctuates within a certain range.
With the increasing number of firings, the deposited metallic particles could form a "bridge" between the trigger and the adjacent electrodes. Raju et al. have observed that the bridge has a very low resistance value [1]. Farrall has observed such a situation that after about 50 shots the trigger resistance was reduced from $6 \mathrm{M} \Omega$ to about $2 \Omega$ and the TVS failed to operate [3]. Consequently the resistance of the dielectric surface will be lower subsequent to higher arc currents.

The TVS-2 has a good performance that the resistance remains a high level in the range 150-200 M $\Omega$ after dozens of firings.

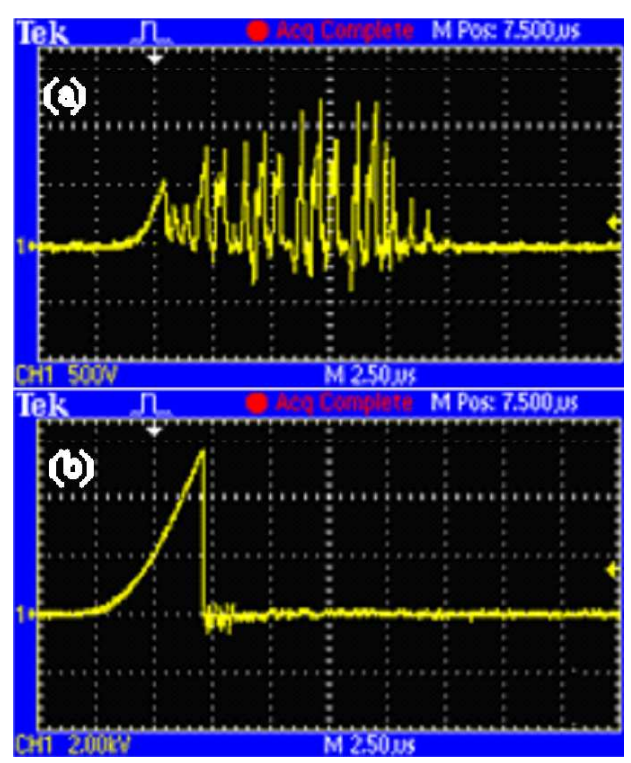

Fig. 4. The typical waveforms of trigger voltage of (a) TVS-1 and (b) TVS-2.

Figure 4 shows typical waveforms of trigger voltage of TVS-1 and TVS-2, respectively. Due to the low resistance, the trigger voltage of TVS- 1 is only $500 \mathrm{~V}$. Comparatively, the trigger voltage of TVS-2 is higher, which is almost $6 \mathrm{kV}$. And the trigger waveform has only a single pulse without any impulsive noise.

The ohmic resistance of a pulse transformer usually lies in the range of several hundred ohms to kilo ohms. In the case of TVS- 1 , the trigger gap resistance is $500 \Omega$, a considerable part of the trigger energy is consumed by the resistance of the pulse transformer already before surface flashover occurs and within every time interval, when the surface arc is extinguished, the trigger voltage rise again. Only a little energy can be transferred into the surface arc. It was reported that the main gap could be fired with minimum trigger energy of $2 \mathrm{~J}$ [4]. And the TVS could be steadily triggered when the trigger energy is sufficient, and would be misfired when the trigger energy is insufficient [5]. In case of TVS-2 the trigger gap resistance is $\mathrm{M} \Omega$, so the energy loss during voltage rise can be neglected. 


\section{Conclusions}

Two surface-breakdown TVS tubes employing a ceramic dielectric coated with a semiconductor layer were experimentally examined. Experimental results show that the variation of the trigger gap resistance is attributed to the deposition of the metal vapor and the evaporation of the semiconductor material. The trigger voltage has the same change tendency as trigger gap resistance.

Raising the trigger gap resistance can improve the performance of TVS effectively, prolonging the service life and reducing the consumed trigger energy in the pulse transformer.

\section{References}

[1] G.R.G. Raju, R. Hackam, F.A. Benson, J. Appl. Phys. 47, 1310 (1976).

[2] G.R.G. Raju, R. Hackam, F.A. Benson, J. Appl. Phys. 48, 1102 (1977).

[3] G.A. Farrall, IEEE Trans. Electron Devices ED-13, 432 (1966).

[4] L.M.J. Vries, G.C. Damstra, IEEE Trans. Power Syst. PWRD-1, 75 (1986).

[5] Z.Y. Zhou, C. Zhao, H.J. Dong, M.F. Liao, Vacuum 44, 58 (2007). 